\title{
SUBDEGREE SUNYAEV-ZEL'DOVICH SIGNAL FROM MULTIFREQUENCY BOOMERANG OBSERVATIONS
}

M. Veneziani ${ }^{1,2,3}$, A. Amblard ${ }^{1}$, A. Cooray ${ }^{1}$, F. Piacentini ${ }^{2}$, D. Pietrobon ${ }^{4,5}$, P. Serra ${ }^{1}$, P. A. R. Ade ${ }^{6}$, J. J. Bock B $^{7,8}$, J. R. Bond ${ }^{9}$, J. Borrill ${ }^{10}$, A. Boscaleri ${ }^{11}$, P. CABella ${ }^{4}$, C. R. Contaldi ${ }^{12}$, B. P. Crill ${ }^{7,8}$, P. De Bernardis ${ }^{2}$, G. De Gasperis ${ }^{4}$, A. De Oliveira-Costa ${ }^{13}$, G. De Troia ${ }^{4}$, G. Di Stefano ${ }^{14}$, K. M. Ganga ${ }^{3}$, E. Hivon ${ }^{15}$, W. C. Jones ${ }^{16}$, T. S. Kisner ${ }^{17}$,

A. E. LAnGE $^{7}$, C. J. MacTavish ${ }^{18}$, S. Masi ${ }^{2}$, P. D. MausKopf ${ }^{6}$, A. Melchiorri ${ }^{2}$, T. E. Montroy ${ }^{17}$, P. Natoli ${ }^{4}$, C. B. Netterfield ${ }^{19}$, E. Pascale ${ }^{19}$, G. Polenta ${ }^{2,20,21}$, S. Ricciardi ${ }^{2,22}$, G. Romeo ${ }^{14}$, J. E. Ruhl ${ }^{17}$, P. Santini ${ }^{2}$, M. Tegmark ${ }^{13}$, AND N. VITTORIO ${ }^{4}$

${ }^{1}$ Center for Cosmology, University of California, Irvine, CA 92697, USA; marcella.veneziani@ roma1.infn.it, amblard@uci.edu

${ }^{2}$ Dipartimento di Fisica, Università di Roma "La Sapienza," Rome, Italy

${ }^{3}$ APC, Université Paris Diderot, 75013 Paris, France

${ }^{4}$ Dipartimento di Fisica, Università di Roma “Tor Vergata," Rome, Italy

${ }^{5}$ Institute of Cosmology and Gravitation, University of Portsmouth, UK

${ }^{6}$ Department of Physics and Astronomy, Cardiff University, Cardiff, UK

7 Jet Propulsion Laboratory, Pasadena, CA 91109, USA

${ }^{8}$ California Institute of Technology, Pasadena, CA 91125, USA

${ }^{9}$ CITA, University of Toronto, Toronto, ON M5S 3H8, Canada

${ }^{10}$ Computational Research Division, LBNL, Berkeley, CA 94720, USA

${ }^{11}$ IFAC-CNR, 50127, Firenze, Italy

12 Theoretical Physics Group, Imperial College, London, UK

${ }^{13}$ Department of Physics, MIT, Cambridge, MA 02139, USA

${ }^{14}$ Istituto Nazionale di Geofisica e Vulcanologia, 00143 Rome, Italy

${ }^{15}$ Institut d'Astrophysique de Paris, 75014 Paris, France

${ }^{16}$ Department of Physics, Princeton University, Princeton, NJ 08544, USA

${ }^{17}$ Case Western Reserve University, Cleveland, OH 44106, USA

${ }^{18}$ Astrophysics Group, Imperial College, London, UK

${ }^{19}$ Physics Department, University of Toronto, Toronto ON, Canada

${ }^{20}$ ASI Science Data Center, c/o ESRIN, 00044 Frascati, Italy

${ }^{21}$ INAF-Osservatorio Astronomico di Roma, Monte Porzio Catone, Italy

${ }^{22}$ Space Sciences Laboratory, UC Berkeley CA, USA

Received 2009 April 28; accepted 2009 July 23; published 2009 August 14

\begin{abstract}
The Sunyaev-Zel'dovich (SZ) effect is the inverse Compton-scattering of cosmic microwave background (CMB) photons by hot electrons in the intervening gas throughout the universe. The effect has a distinct spectral signature that allows its separation from other signals in multifrequency CMB data sets. Using CMB anisotropies measured at three frequencies by the BOOMERANG 2003 flight we constrain SZ fluctuations in the 10 arcmin to $1 \mathrm{deg}$ angular range. Propagating errors and potential systematic effects through simulations, we obtain an overall upper limit of $15.3 \mu \mathrm{K}(2 \sigma)$ for rms SZ fluctuations in a broad bin between multipoles of 250 and 1200 at the Rayleigh-Jeans (RJ) end of the spectrum. The resulting upper limit on the local universe normalization of the density perturbations with BOOMERANG SZ data alone is $\sigma_{8}^{\mathrm{SZ}}<1.14$ at the $95 \%$ confidence level. When combined with other CMB anisotropy and SZ measurements, we find $\sigma_{8}^{\mathrm{SZ}}<0.92$ (95\% c.l.).

Key words: cosmic microwave background - cosmological parameters - cosmology: observations - large-scale structure of universe
\end{abstract}

\section{INTRODUCTION}

The Sunyaev-Zel'dovich (SZ) effect (Sunyaev et al. 1972) is the scattering of cosmic microwave background (CMB) photons by electrons in the intervening gas throughout the universe. The SZ effect is generally subdivided into two subcomponents: the kinetic effect due to the bulk motion and the thermal effect due to energy transfer from hot electrons. The latter is expected to be the largest modification to the background temperature during photon transit from the last scattering surface. We will concentrate on it hereafter and refer to it as the SZ signal. The strongest SZ perturbations to the CMB temperature are dominated by the scattering of photons via hot electrons in massive galaxy clusters (Komatsu \& Kitayama 1999; Springel et al. 2001; Cooray 2000; Molnar \& Birkinshaw 2000; Seljak et al. 2001; Sadeh \& Rephaeli 2004), but there could also be an SZ signal from reionization (Oh et al. 2003). The integrated SZ angular power spectrum is now a known probe of the amplitude of density perturbations, $\sigma_{8}$ (Komatsu et al. 2002; Bond et al. 2005).

The SZ effect has been clearly imaged toward individual galaxy clusters (Grego et al. 2000; Carlstrom et al. 2002; Jones et al. 1993) and has been used for a variety of applications, including a measurement of the $\mathrm{CMB}$ temperature at the redshifts of Coma and A2163 (Battistelli et al. 2002). However, the amplitude of the SZ power spectrum at arcminute angular scales, generated from unresolved galaxy clusters, is still not well established with differences at the $2 \sigma$ level from a variety of detections and limits (Sievers et al. 2009; Reichardt et al. 2008; Dawson et al. 2006; Sharp et al. 2009; Friedman et al. 2009; Sayers et al. 2008). Existing SZ anisotropy measurements are restricted to observations with a narrow frequency coverage and to small areas on the sky. The differences could be a combination of foreground contamination and large non-Gaussian variance 
of the SZ signal (Cooray 2001). Also, no constraints on the SZ signal exist at tens of arcminute scales where primary CMB fluctuations dominate.

A clean separation of the SZ anisotropies from primordial $\mathrm{CMB}$ is possible due to the fact that the SZ signal has a distinct frequency spectrum from the $2.7 \mathrm{~K}$ blackbody spectrum (Cooray et al. 2000). The spectral difference arises as inverse-Compton scattering leads to, on average, a net energy gain for the CMB photons and the scattered photons move from the low frequency Rayleigh-Jeans (RJ) tail to high frequencies (Sunyaev et al. 1972). The SZ sky is colder than the CMB at low frequencies and hotter than the $\mathrm{CMB}$ at high frequencies with no difference at about a frequency of $217 \mathrm{GHz}$. A potential detection of the largescale structure SZ fluctuations is then aided by observations across the SZ null from the negative side to the positive side.

A data set of the form needed for a study of the large-scale structure SZ effect is provided by the 2003 flight of the balloonborne BOOMERANG experiment (Masi et al. 2006). This instrument derives directly from the BOOMERANG payload that was flown in 1998 and resulted in first high signal-tonoise maps of the CMB anisotropy with subhorizon resolution (de Bernardis et al. 2000). The instrument was launched by NASA on 2003 January 6 from Williams Field near McMurdo Station, in Antarctica. The flight lasted a total of $311 \mathrm{hr}$ until 2003 January 21 and $119 \mathrm{hr}$ of this observing period were devoted to scanning a deep survey region. The remaining time was spent on scanning a larger shallow survey and a section of the Galactic plane. Here, we concentrate on a search for SZ effect in the central deep field over $100 \mathrm{deg}^{2}$ with the highest signal-to-noise ratio.

In this Letter, we report the first statistical limits of the SZ signal at subdegree angular scales at these wavelengths. The discussion is organized as follows: in Section 2 we detail our approach to extract the SZ signal from the multifrequency BOOMERANG data set; in Section 3 we detail our simulations used to estimate statistical and systematic uncertainties; and in Section 4 we present our results.

\section{CMB AND FOREGROUND REMOVAL}

To separate the SZ signal from all other sources of anisotropies, we adopted a technique well known in the literature for removing foregrounds from the CMB anisotropies (Tegmark \& Efstathiou 1996; Tegmark et al. 2003; Amblard et al. 2007). In our case, instead of recovering the primordial CMB signal, we recover SZ fluctuations by minimizing the covariance relative to the SZ frequency dependence, and treat primordial fluctuations as another source of noise. In the remainder of this Letter, we will refer to "foregrounds" as all the additional emissions (CMB, Galactic dust, far-IR sources or FIRB, radio point sources) to the SZ effect.

The power spectrum of the SZ can be obtained from a weighted mean of the power spectra at different frequencies: $C_{l}^{\mathrm{SZ}}=\mathbf{w}^{T} \mathbf{C w}$ (Cooray et al. 2000). The weights $w_{l}^{i}$ at each frequency $i$ and multipole $\ell$ can be obtained by minimizing the covariance of data multipole moments $\mathbf{C} \equiv\left\langle\hat{a}_{l m}^{i} \hat{a}_{l m}^{j}\right\rangle$ subject to the constraint that SZ estimation is unbiased $\left(\sum_{i} w\left(v_{i}\right)=1\right)$.

In the case of BOOMERANG maps, each frequency channel consists of several detectors. We distinguish individual detectors with the indices $(\alpha, \beta)$, while $(i, j)$ are indices for the frequency channels. In order to minimize instrumental noise more aggressively, we compute the covariance matrix of the signal by averaging all the combinations of cross-spectra between

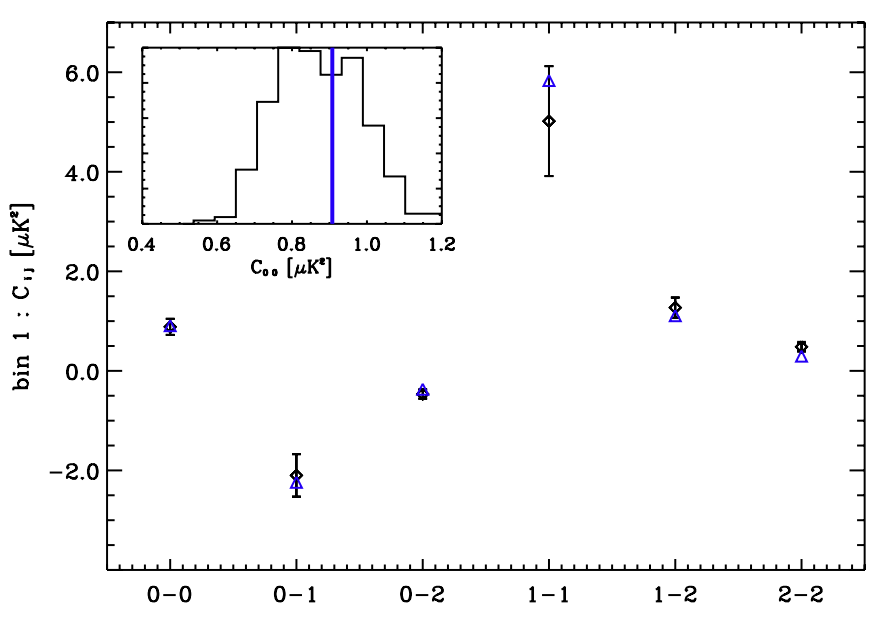

Figure 1. Elements of the matrix $C_{i j}$ (Equation (1)) for the data (blue triangle) and simulations in $(\mu \mathrm{K})^{2}$ RJ units in the first multipole bin $(250<\ell<450)$. The six independent points of the $C_{i j}$ matrix correspond to correlation between frequencies labeled 0,1 , and 2 for $145 \mathrm{GHz}, 245 \mathrm{GHz}$, and $345 \mathrm{GHz}$, respectively. We show two sets of distributions for simulations. The smaller distribution takes into account only instrumental noise and cosmic variance. The larger distribution includes also primary $\mathrm{CMB}$ and foregrounds by taking the rms of three different amplitudes for the components (see text). In the case where only one error bar is visible, the rms from foreground is smaller than the instrumental noise and cosmic variance. The inserted plot shows the distribution of $C_{00}$ with the data value inserted as a vertical blue line.

different detectors and ignoring the auto-spectra of the same detector. The contribution of the correlated noise between two different detectors is taken into account in the simulations and removed as part of a residual contribution to the SZ signal.

We construct the binned covariance matrix in multipole $\ell$ bin $b$ as

$$
\mathbf{C}_{i j}=\sum_{l \in b, m} \sum_{\alpha, \beta} \frac{\left\langle a_{l m}^{i, \alpha} a_{l m}^{j, \beta^{\star}}\right\rangle}{s\left(v_{i}\right) s\left(v_{j}\right) b_{l}^{i, \alpha} b_{l}^{j, \beta}} \quad \text { with } \alpha \neq \beta, \text { if } i=j,
$$

where $s\left(v_{i}\right)$ is the SZ frequency dependence at each of the BOOMERANG frequency bands relative to CMB with $s(v)=$ $2-(x / 2) \operatorname{coth}(x / 2), x=h v / k T_{\mathrm{CMB}} \approx v / 56.8 \mathrm{GHz}$, and $b_{l}^{i, \alpha}$ is the measured beam window function for the detector $\alpha$ in channel $i$. Note that with the definition above, in the RJ limit $s(v) \rightarrow 1$ so that $C_{l}^{\mathrm{SZ}}\left(v, v^{\prime}\right)=s(v) s\left(v^{\prime}\right) C_{l}^{\mathrm{SZ}}$ where $C_{l}^{\mathrm{SZ}}$ is the $\mathrm{SZ}$ anisotropy power spectrum in the RJ limit. The covariance matrix $\mathrm{C}_{i j}$ is required to be invertible and positive definite. We numerically check this both in data and simulations. In Figure 1 we show the covariance matrix from data and compare it to simulations described below.

Using the data covariance matrix, the optimal weights for the SZ reconstruction are

$$
\mathbf{w}=\frac{\mathbf{C}^{-1} \mathbf{e}}{\mathbf{e}^{T} \mathbf{C}^{-1} \mathbf{e}}
$$

where $\mathbf{e}$ is a unit vector, $e\left(v_{i}\right)=1$. The BOOMERANG channels consist of eight polarization-sensitive bolometers at 145 $\mathrm{GHz}$, and four spider-web bolometers at each of $245 \mathrm{GHz}$ and $345 \mathrm{GHz}$ channels. We make use of data from all these detectors except two detectors that were known in prior studies to be dominated by detector noise (245X and 345Z) (Masi et al. 2006) and two detectors with a significantly higher noise than the others (145Z2 and 345Y), leaving us with seven detectors at 145 , three at 245 , and two at $345 \mathrm{GHz}$. 
We use the spectral response of each band as measured in the lab with subpercent accuracy. From these bands we derived the values of $(0.49722,-0.21646,-1.01643)$ for $s(v)$ at 145,245 , and $345 \mathrm{GHz}$, respectively. These bands provide ideal frequency coverage for an SZ study with channels in the SZ decrement, near the null, and the increment, respectively. The measured FWHM of the beams is $11.5,8.5$, and 9.1 arcmin for the 145,245 , and $345 \mathrm{GHz}$ channel, respectively (Jones et al. 2006). These values include a 2.4 arcmin pointing jitter. The beams' window functions $b_{l}^{i, \alpha}$ are in fact numerically derived from physical optics simulations, combined with a Gaussian pointing jitter.

Similar to prior studies with BOOMERANG data, we produced CMB temperature anisotropy (T) maps using the Italian analysis pipeline (Masi et al. 2006) and the TT power spectra with the MASTER method (Hivon et al. 2002). To remove excess atmospheric noise, we filtered out time-ordered data in each of the detectors in frequency space below $200 \mathrm{mHz}$. This results in a damping of power at angular scales above 1.2. The effect of this filter, of the scanning strategy, of the sky coverage, and of the pixelization results in an effective window function. This window function is estimated by projecting signal-only sky simulation into detector time-streams. To do that we used 10 sky simulations of the SZ signal, obtained from White (2003) with different normalizations and initial conditions. These timestreams are then analyzed with our pipeline and the resulting angular power spectrum is compared to the power spectrum of the input sky signal to obtain the window function. The 10 different simulations result in slightly different window functions. The scatter in the window function is included in the final error estimate of the SZ signal.

\section{SIMULATIONS}

Monte Carlo simulations were used in order to estimate the residual signal from correlated detector noise, primary CMB, and foregrounds that is detected as an SZ signal at the end. In fact, when we minimize the covariance in the data to extract the SZ, the optimal combination does not remove perfectly the other signals. This is particularly true with just three channels, and is expected to improve with more channels. We also use simulations to estimate final uncertainties in the SZ signal.

We generate 200 time-stream simulations for each one of the 12 detectors with a combination of sky signal simulations that were projected into a time-line using the BOOMERANG pointing, and a random noise realization with variance consistent with data. As the covariance matrix $\mathrm{C}_{i j}$ of Equation (1) is built using the cross-spectra between different detectors, the noise realizations include also correlated noise between different detectors. For $145 \mathrm{GHz}$ the noise correlation spectra are reported in Table 7 of Masi et al. (2006) and they are below $3 \%$ for the detectors used in the analysis. For the 245 and $345 \mathrm{GHz}$ we measured the noise correlation spectra removing the optimal signal map from the time-ordered data. The correlations are at most $6 \%$ for the detectors used in the analysis.

In addition to 200 realizations of primary CMB, the sky signal simulations are computed with Galactic dust, far-Infrared background (FIRB) sources, and radio point sources and combined with BOOMERANG band passes. The Galactic dust for the observed field is described with model 8 from Finkbeiner et al. (1999), while FIRB and point sources were obtained from the Planck sky model (PSM; Leach et al. 2008; J. Delabrouille 2009, in preparation).

The amplitude of each component in the data was estimated with a Monte Carlo Markov Chain for the three frequencies together. We compared the measured temperature angular power spectrum with a linear combination of CMB, dust, FIRB, and point sources and fitted the amplitude coefficients $p_{\gamma}$ of each component $\gamma$ such that $C_{l}^{\text {tot }}=\sum_{\gamma} p_{\gamma} C_{l}^{\gamma}+N_{l}$, where $N_{l}$ is detector noise. The CMB amplitude of the predicted model is the same as we find in the data with $p_{\mathrm{CMB}}=1.01 \pm 0.04$. We find $p_{\text {dust }}=4.6 \pm 0.8$, larger than predicted by model 8 of Finkbeiner et al. (1999) and $p_{\text {radio }}=0.76 \pm 0.07$ for radio point sources, smaller than predicted by de Zotti et al. (2005) counts used in PSM, but in agreement with Friedman et al. (2009). The amplitude of the FIRB remains unconstrained with $0<p_{\text {FIRB }}<0.7(1 \sigma)$.

To take the uncertainties in the amplitudes of foregrounds into account, we ran three sets of 200 simulations. In the first set we leave the level of foregrounds as the models predict ( $p_{\alpha}=1$ for each of CMB, dust, FIRB, and radio sources). In the second case we set $p_{\text {dust }}=4.6, p_{\text {FIRB }}=0$, and $p_{\text {radio }}=0.76$. In the third case, we change $p_{\text {FIRB }}=0.7$, while keeping the rest of the parameters as in the second case. We note that these simulations do not include the SZ signal as we are reconstructing the $\mathrm{SZ}$ under the assumption of a zero signal. This does not bias the procedure since the optimal weights from Equation (1) are independent of the exact amplitude of the large-scale SZ effect.

The simulated time-lines have been analyzed with the same pipeline as used for the data. This results in three sets of Monte Carlo binned power spectra for the SZ $C_{b \mathrm{MC}}^{\mathrm{SZ}}$, which should, in principle, be zero since the SZ signal is not included in the simulations. From the distribution of each set of $C_{b \mathrm{MC}}^{\mathrm{SZ}}$ we derived (1) a bias in the $\mathrm{SZ}$ binned power spectrum from $\left\langle C_{b \mathrm{MC}}^{\mathrm{SZ}}\right\rangle$ and (2) the bin-to-bin covariance matrix due to statistical noise and sampling variance of the $\mathrm{CMB}$,

$$
\mathcal{C}_{b b^{\prime}}=\left\langle\left(C_{b \mathrm{MC}}^{\mathrm{SZ}}-\left\langle C_{b \mathrm{MC}}^{\mathrm{SZ}}\right)\right) \times\left(C_{b^{\prime} \mathrm{MC}}^{\mathrm{SZ}}-\left\langle C_{b^{\prime} \mathrm{MC}}^{\mathrm{SZ}}\right)\right)\right\rangle .
$$

The error bars on the angular power spectrum are given by $\Delta C_{b}^{\mathrm{SZ}}=\sqrt{\mathcal{C}_{b b}}$. In Table 1 , we quote the average value of the bias from the three sets in our residual amplitude and add the average of the dispersion of these residuals as an additional error (Table 1 foreground error).

These foreground errors are combined with the beam errors, which are estimated again through Monte Carlo simulations. The calibration uncertainties of the time-lines were also included through Monte Carlo simulations. They are at most 2\%, $8 \%$, and $13 \%$ for the $145 \mathrm{GHz}, 245 \mathrm{GHz}$, and $345 \mathrm{GHz}$, respectively, leading to an error of $4 \%, 16 \%$ and $26 \%$ on the temperature angular power spectra at each of the three frequencies. All the uncertainties listed above are added in quadrature for the final SZ band-power uncertainty.

\section{SZ POWER SPECTRUM ESTIMATE}

In Table 1 we list the values we obtain for the three multipole bins between $\ell=250$ and 1200 . We also list the residual level from each foreground component, including detector noise, and the error associated with various uncertainties as described above. As tabulated, the biggest contamination to SZ detection comes from instrumental noise at the largest angular scales, while the FIRB dominates the contamination at the smallest angular scales probed by the experiment. Radio point sources generate negligible confusion, primarily because at these high frequencies radio sources produce a weaker background compared to the dusty galaxies making the FIRB. In accounting for foreground contamination in the SZ estimate, we have taken a conservative approach here allowing for all 
Table 1

SZ Power Spectrum Estimates

\begin{tabular}{|c|c|c|c|}
\hline$\ell$-range & $\begin{array}{c}\text { Bin } 1 \\
250-450\end{array}$ & $\begin{array}{c}\text { Bin } 2 \\
450-700 \\
\end{array}$ & $\begin{array}{c}\text { Bin } 3 \\
700-1200 \\
\end{array}$ \\
\hline \multicolumn{4}{|c|}{ Optimal weights } \\
\hline$w_{145 \mathrm{GHz}}$ & 0.9323 & 0.8514 & 0.7289 \\
\hline$w_{245 \mathrm{GHz}}$ & 0.4193 & 0.3771 & 0.3002 \\
\hline$w_{345 \mathrm{GHz}}$ & -0.3515 & -0.2285 & -0.0292 \\
\hline Raw SZ & 236 & 164 & 538 \\
\hline \multicolumn{4}{|c|}{ Residuals $^{\mathrm{a}}$} \\
\hline CMB & 53 & 36 & 70 \\
\hline Instr. noise & 92 & 12 & -95 \\
\hline Galactic dust & 68 & 82 & 138 \\
\hline FIRB & 44 & 81 & 195 \\
\hline Radio sources & 3 & 7 & 58 \\
\hline Total residual & 247 & 202 & 338 \\
\hline \multicolumn{4}{|c|}{ SZ Band Power Uncertainties ${ }^{\mathrm{b}}$} \\
\hline Instr. noise & 154 & 116 & 280 \\
\hline Foregrounds & 37 & 79 & 145 \\
\hline Beam & 3 & 5 & 44 \\
\hline Calibration & 121 & 77 & 63 \\
\hline Transfer func. & 2 & 3 & 11 \\
\hline Cosmic \& NG $\operatorname{Var}^{\mathrm{c}}$ & 7 & 6 & 4 \\
\hline Final SZ Band Power & $-11 \pm 199$ & $-38 \pm 160$ & $200 \pm 325$ \\
\hline
\end{tabular}

Notes. The weights and Raw SZ designate the weight vectors for each multipole bin and the SZ power spectrum respectively with both as measured from data. Except in the case of weights $\mathbf{w}$, the values are tabulated in units of $\mu \mathrm{K}^{2}$ for the $\mathrm{SZ}$ angular power spectrum $l^{2} C_{l} / 2 \pi$ at the RJ end of the frequency spectrum.

a The residuals are the average spectra measured on our SZ-free simulations and represent our bias. The total residual is different from the sum of the partial residuals due to small $(<10 \%)$ random correlation between components.

$\mathrm{b}$ The uncertainties are the dispersion measured with our simulations. The final SZ spectrum values are corrected for the noise and foreground bias with the dispersion error from simulations.

c Assuming the WMAP team's SZ power spectrum with $\sigma_{8}=0.95$, the $2 \sigma$ upper limit we derived from all SZ data. The calculation for the Non-Gaussian (NG) covariance makes use of the same halo model as used for this power spectrum.

components. An aggressive approach with the assumption of no FIRB leads to a marginal detection of an SZ signal especially in the third bin. Though the amplitude of FIRB fluctuations at $350 \mathrm{GHz}$ is uncertain and we have based our model on the PSM, we do not consider an SZ detection with a no FIRB assumption to be realistic.

The binned SZ power spectrum limits at the RJ end of the frequency spectrum are shown in Figure 2, where we plot the $68 \%$ confidence level limit for three bins between multipoles of 250 and 1200. In estimating the final SZ band power uncertainty, we also include the usual Gaussian cosmic variance and the extra covariance from the non-Gaussian nature of the SZ power spectrum (Cooray 2001). This covariance is calculated assuming $\sigma_{8}^{\mathrm{SZ}}=0.95$ and making use of the same halo model as the one used by the WMAP team's SZ model and shown with a solid line in Figure 2 (Komatsu et al. 2002). Within this model, we study the cosmological implications of our limit on the SZ fluctuations, using a MCMC package (Lewis \& Bridle 2002) to constrain the amplitude of fluctuations. For reference to numerical simulations, with a dashed line, we also show the average SZ signal and the scatter from a set of ten simulations at $\sigma_{8}=1$ from White (2003).

In addition to three bins shown in Figure 2, we also combine the estimation of BOOMERANG SZ power spectrum to a single

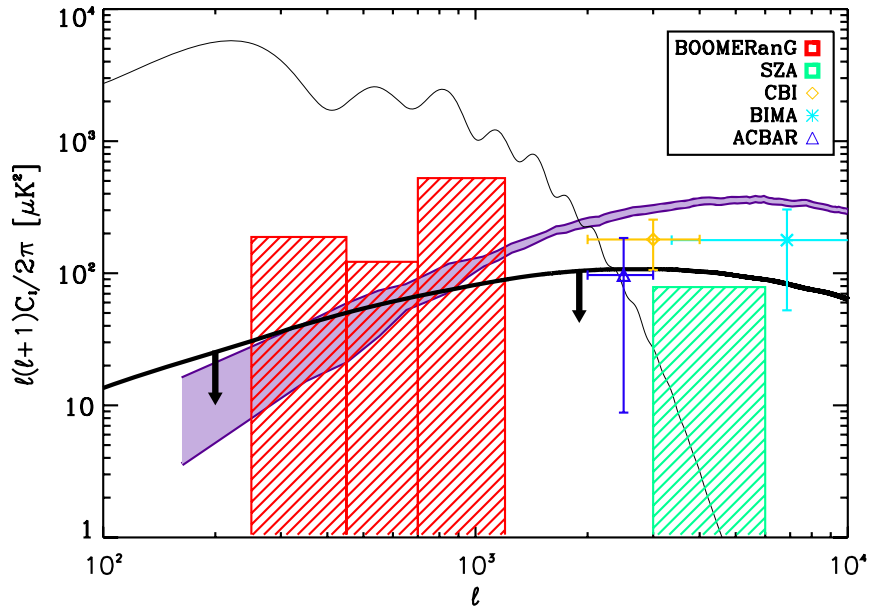

Figure 2. Angular power spectrum of SZ anisotropies at the RJ end of the frequency spectrum. We show the $68 \%$ confidence upper limits from the BOOMERANG data in hashed columns to the left and the reported upper limit at the $65 \%$ confidence level from the SZA experiment to the right. The data points from CBI, BIMA, and ACBAR experiments are also shown (see text for details). We correct the ACBAR point to RJ end of the frequencies. The lines show two theory predictions for SZ fluctuations: the curved region is a prediction based on results from numerical simulations with $\sigma_{8}^{\mathrm{SZ}}=1$ and the solid line is the same model as used by the WMAP team but scaled to $\sigma_{8}^{\mathrm{SZ}}=0.95$, the $2 \sigma$ upper limit from all SZ data. The limit from BOOMERANG SZ data alone is $\sigma_{8}^{\mathrm{SZ}}<1.14$ (95\% c.1.)

broad bin of $250<\ell<1200$. We find an upper limit of $234 \mu \mathrm{K}^{2}$ in $l(l+1) C_{l} / 2 \pi$ at the $95 \%$ confidence level. Previous analytical calculations have shown that $C_{l}^{\mathrm{SZ}} \propto\left(\sigma_{8}^{\mathrm{SZ}}\right)^{7}\left(\Omega_{b} h\right)^{2}$ (Seljak et al. 2001), where we separate $\sigma_{8}^{\mathrm{SZ}}$ associated with SZ from the primordial normalization $\sigma_{8}$. The amplitude constraint from BOOMERANG SZ data alone is $\sigma_{8}^{\mathrm{SZ}}<1.14$ at $95 \%$ confidence level.

In Figure 2, we also compare our upper limits with results on SZ fluctuations in the literature, including CBI (Sievers et al. 2009), BIMA (Dawson et al. 2006), and ACBAR (Reichardt et al. 2008). We scale the ACBAR value from $150 \mathrm{GHz}$ to the RJ end of the spectrum for easy comparison with all other results. We also fit jointly the combined WMAP five-year (Komatsu et al. 2009), ACBAR (Reichardt et al. 2008), and CBI (Sievers et al. 2009) data together with SZ upper limits from BOOMERANG and SZA. We use the same analytical halo model with $\sigma_{8}^{\mathrm{SZ}}=0.95$ to include an extra uncertainty associated with non-Gaussian covariance in each of these measurements; these, however, make only a minor difference except in the case of BIMA where the smaller area surveyed increase the importance of non-Gaussianities. Marginalizing over all other cosmological parameters in the $\Lambda \mathrm{CDM}$ model, we find $\sigma_{8}^{\mathrm{SZ}}<0.92$ at $95 \%$ confidence level $\left(\sigma_{8}^{\mathrm{SZ}}<0.71\right.$ at $\left.1 \sigma\right)$. This $\mathrm{SZ}$ derived amplitude is fully consistent with WMAP five-year result with $\sigma_{8}=0.81 \pm 0.02$ (Komatsu et al. 2009). While it has been claimed in the past that the SZ derived $\sigma_{8}$ is higher than the value derived from the CMB, we do not find this is the case with the BOOMERANG data.

The next opportunities to perform a multifrequency analysis similar to ours will be with Planck and OLIMPO (Masi et al. 2008). Both these experiments include multiple bands at high frequencies where the SZ is positive. As we have found that only one channel above the SZ null frequency is not adequate to separate both CMB and FIRB from SZ fluctuations, with several high frequency channels, these upcoming $\mathrm{CMB}$ experiments should be able to measure and separate FIRB 
more accurately than we were able to with just one channel at $350 \mathrm{GHz}$.

We gratefully acknowledge support from NSF CAREER AST-0645427 at UCI. We also acknowledge support from the Italian Space Agency (contracts I/087/06/0 and I/016/ 07/0), and from Programma Nazionale Ricerche in Antartide. The authors acknowledge the use of the Planck Sky Model, developed by the Component Separation Working Group (WG2) of the Planck Collaboration.

\section{REFERENCES}

Amblard, A., Cooray, A., \& Kaplinghat, M. 2007, PRD, 75, 083508 Battistelli, E. S., et al. 2002, ApJ, 580, L101

Bond, J. R., et al. 2005, ApJ, 626, 12

Carlstrom, J. E., Holder, G. P., \& Reese, E. D. 2002, ARA\&A, 40, 643

Cooray, A. 2000, Phys. Rev. D, 62, 103506

Cooray, A. 2001, Phys. Rev. D, 64, 063514

Cooray, A., Hu, W., \& Tegmark, M. 2000, ApJ, 540, 1

Dawson, K. S., et al. 2006, ApJ, 647, 13

de Bernardis, P., et al. 2000, Nature, 404, 955

de Zotti, G., et al. 2005, A\&A, 431, 893

Finkbeiner, D. P., Davis, M., \& Schlegel, D. J. 1999, ApJ, 524, 867
Friedman, R. B., et al. 2009, ApJ, 700, L107

Greggo, L., et al. 2000, ApJ, 539, 39

Hivon, E., et al. 2002, ApJ, 567, 2

Jones, M., et al. 1993, Nature, 365, 320

Jones, W. C., et al. 2006, ApJ, 647, 823

Komatsu, E., \& Kitayama, T. 1999, ApJ, 526, L1

Komatsu, E., \& Seljak, U. 2002, MNRAS, 336, 1256

Komatsu, E., et al. 2009, ApJS, 180, 330

Leach, S. M., et al. 2008, A\&A, 491, 597

Lewis, A., \& Bridle, S. 2002, PRD, 66, 103511

Masi, S., et al. 2006, A\&A, 458, 687

Masi, S., et al. 2008, Mem. Soc. Astron. Ital., 79, 887

Molnar, S., \& Birkinshaw, M. 2000, ApJ, 537, 542

Oh, S.-P., Cooray, A., \& Kamionkowski, 2003, MNRAS, 342, L20

Reichardt, C. L., et al. 2008, ApJ, 694, 1200

Sadeh, S., \& Rephaeli, Y. 2004, NA, 9, 373

Sayers, J., et al. 2008, ApJ, 690, 1597

Seljak, U., Burwell, J., \& Pen, Ue-Li 2001, Phys. Rev. D, 63, 063001

Sharp, M. K., et al. 2009, ApJ, submitted (arXiv:0901.4342)

Sievers, J. L., et al. 2009, arXiv:0901.4540

Springel, V., White, M., \& Hernquist, L. 2001, ApJ, 549, 681

Sunyaev, R. A., \& Zeldovich, Ya. B. 1972, Comments Astrophys. Space Phys., 4, 173

Tegmark, M., de Oliveira-Costa, A., \& Hamilton, A. 2003, Phys. Rev. D, 68, 123523

Tegmark, M., \& Efstathiou, G. 1996, MNRAS, 281, 1297

White, M. 2003, ApJ, 597, 650 